\title{
A REVIEW OF ROBOCASTING TECHNOLOGY
}

\author{
JOSEPH CESARANO III
}

Direct Fabrication Technologies Department, Sandia National Laboratories, Albuquerque, NM 87185-1349, jcesara@sandia.gov

\begin{abstract}
Robocasting is a freeform fabrication technique for dense ceramics and composites that is based on layer-wise deposition of highly loaded colloidal slurries. The process is essentially binderless with less than $1 \%$ organics and parts can be fabricated, dried, and completely sintered in less than 24 hours. This review will highlight materials developments for structural applications and modelling of slurry flow. Fabrication of preforms for alumina / metal composites will be discussed as well as techniques for multimaterial deposition in both graded structures and discrete placement of fugitive materials.
\end{abstract}

\section{INTRODUCTION}

Robocasting is being developed at Sandia National Laboratories for the freeform fabrication of ceramics and composites. Robocasting uses robotics for the layerwise deposition of ceramic slurries through an orifice. Orifice openings can range from a couple of millimeters to tenths of millimeters. The process is based on the extrusion of highly loaded ceramic slurries that are typically $50-65 \mathrm{vol} . \%$ ceramic powder, $<1$ vol.\% organic additives, and $35-50 \mathrm{vol} . \%$ volatile solvent (usually water). Since binder burnout is not an issue, a dense ceramic part may be freeformed, dried, and sintered in less than 24 hours. Robocasting is described in detail in reference [1].

In general, a robocasting slurry must meet three criteria: 1) it must be pseudoplastic enough to flow through a small orifice at modest shear rates; 2 ) it must set-up into a nonflowable mass upon dispensing; and 3) it must be able to "accept" multiple layers without defects to form a uniform mass. Probably the most unique and interesting aspect of robocasting is the process by which the flowing pseudoplastic slurry transforms into a solid-like mass after deposition. In contrast to gel casting and other freeform fabrication techniques, robocasting does not require organic polymerization reactions or solidification of a polymeric melt for the solid transformation. On the contrary, in order to maintain structural integrity while building a component, robocasting relies on the rheology of the deposited slurry and on partial drying of the individual layers. This is explained below.

Typical ceramic powder slurries have an average particle size on the order of several microns and posses a relatively monosized distribution. Ceramic powders with this character, that are dried from a dispersed slurry, typically pack into a consolidated structure that is approximately $65 \%$ of the theoretical density. For robocasting, the character of flowable slurries with solids loadings just below the consolidated density is crucially important. Figure IA depicts schematically the behavior of a typical dispersed alumina powder slurry. At low solids loadings, dispersed slurries have very low viscosity and are rheologically Newtonian. Around 40 volume percent solids, the slurries begin to show pseudoplastic shear-thinning behavior even though the viscosity is still relatively low. As the solids content approaches 60 volume percent, inter-particle interactions and inter-particle collisions become dominant; viscosity begins to increase appreciably and the rheological behavior becomes highly shearthinning. At approximately 63 volume percent solids, particle mobility becomes restricted and the slurry locks up into a dilatant mass. Therefore, it is desirable to robocast with slurries that 
have solids loadings approaching the dilatant transition so that with minimal drying a robocasted layer becomes structurally sound and a foundation upon which more layers may be deposited.

Figure 1B schematically depicts how the pseudoplastic to dilatant transition must closely follow the build rate in order to maintain structural integrity for thick parts. Therefore, the drying kinetics of the freshly deposited beads determine the optimum build parameters. Typically, parts are built upon a platform heated between 30 and 60 degrees Celsius to assist the pseudoplastic to dilatant transition. For thick parts it is necessary to incorporate an additional heat source above the build platform. From Fig. 1B it is evident that if the drying rate is too slow, the pseudoplastic to dilatant transition is delayed and accumulated weight from several layers eventually surpasses the yield stress of the pseudoplastic layers. This condition can induce slumping and the creation of nonuniform walls. Conversely, if the drying rate is too fast, warping, cracking, and delamination may occur.

In general, proper robocasting requires a synergistic control of the : 1) percent solids in the ceramic powder slurry, 2) viscosity and rheology of the slurry, 3) dispensing rate of the slurry through the orifice, 4) drying kinetics of the dispensed bead of slurry, and 5) computer code for optimal machine instructions. When a proper balance of these variables is achieved, robocasting can be used to make intricate ceramic bodies that sinter into relatively strong, dense and defect free parts.

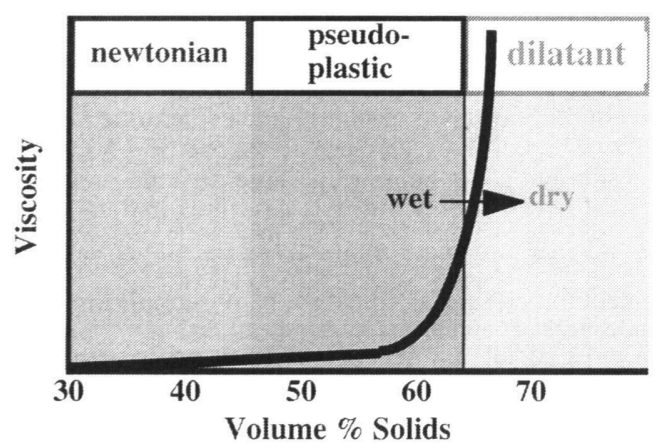

A

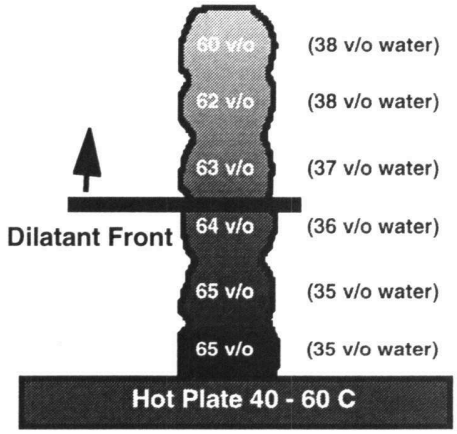

B

Figure 1: A) A schematic showing the typical viscosity versus volume percent solids behavior for dispersed alumina slurries. For optimal robocasting, work close to the dilatant transition. B) A schematic showing how a part "solidifies" during building through a pseudoplastic / dilatant rheological transition.

\section{EXPERIMENTAL}

The slurries discussed throughout this paper were made and deposited in accordance with references [1] and [2]. The robotic slides used for the $\mathrm{X}, \mathrm{Y}, \mathrm{Z}$ and dispensing axes were purchased from Velmex, Inc. The slides were controlled with servo motors and controllers from Galil Motion Control, Inc. The three-dimensional modelling of bead flow was based on GOMA finite element calculations developed by Thomas A. Baer at Sandia National Laboratories. 


\section{RECENT DEVELOPMENTS AND DISCUSSION}

Our current work is now being directed at characterizing and modelling the robocasting process in an effort to optimize build parameters for improved control and tolerance of part fabrication. Also, we are exploring new uses and opportunities related to materials fabrication to exploit the versatility of robocasting for nontraditional manufacturing methods and for multimaterial fabrication.

- Modelling: We have already shown that robocast alumina parts have densities and strengths comparable to alumina processed more traditionally [3]. However, there is a lot of room for improvement when looking at the dimensional tolerance and surface finish that robocasting provides. With that in mind, we are attempting to model the flow of beads of slurry to derive a knowledge-base for the exact shape of deposited beads as a function of build parameters. It is fortunate that it has already been determined that as the freshly deposited bead undergoes a dilatant transition into a solid-like mass, there is a minimal change in shape. There is appreciable flow and shape change as the slurry is being sheared during deposition. However, King et al [4] have shown with non-contact laser profilometry that alumina beads have minimal dimensional change after deposition. In fact, the entire dilatant transition takes approximately one minute to occur even at room temperature. With heat the transition occurs more rapidly.

Figure 2 is a schematic showing some results from the three-dimensional modelling of bead flow. The code is currently based on finite element analysis of a Newtonian slurry. Even though pseudoplasticity has not yet been taken into account, the bead shape predictions are very good for deposition onto a moving platform. However, the actual image in Figure 3C shows that the deposition behavior into a previously dispensed bead is very different than deposition onto a moving platform. The freshly dispensed bead wets the previously deposited bead. Therefore, the fresh slurry is pulled down and fills space over the entire curved top surface of the previously deposited bead. Also, the leading edge of the fresh slurry is now pulled even to the front of the orifice instead of lagging behind (Fig. 3A). This experiment shows the space filling behavior of slurries, that is beneficial for the fabrication of defect free parts. However, it also shows that the bead flow model will have to include calculations for deposition onto curved wetting surfaces in order to accurately predict part dimensions and tolerances.

- Preforms for Alumina / Metal Composites: In addition to fabricating single material parts, robocasting may have utility for the manufacture of intricate preforms for the fabrication of ceramic / metal joining composites. By robocasting various crosshatch patterns, intricate structures may be fabricated that can not obviously be manufactured by traditional fabrication techniques. Figure 4A shows a cross-section of a robocast alumina part fabricated with regions of closed porosity as well as open voids with large undercuts. This type of structure when infiltrated with a metal forms a mechanically bonded ceramic to metal join that has a graded composition on a macroscale. Figure 4B shows the cross-section of a similar preform that was infiltrated with an active metal (TiCuSil). This part not only showed exemplary bonding without cracking but was subsequently used as a platform upon which LENS [5] processed stainless steel was freeformed. In finality, a structurally sound crack-free part was fabricated that transitioned from $100 \%$ alumina to $100 \%$ stainless steel. Additionally, the part was mostly freeformed. The TiCuSil metal used to fabricate the composite in Fig. 4B is probably prohibitively expensive for any widespread application. Therefore, a method for infiltrating aluminum metal into a robocast alumina preform was developed. Figure $4 \mathrm{C}$ shows a crosssection of a structurally sound alumina / aluminum part that is macroscopically graded with some mechanical interlocking. 

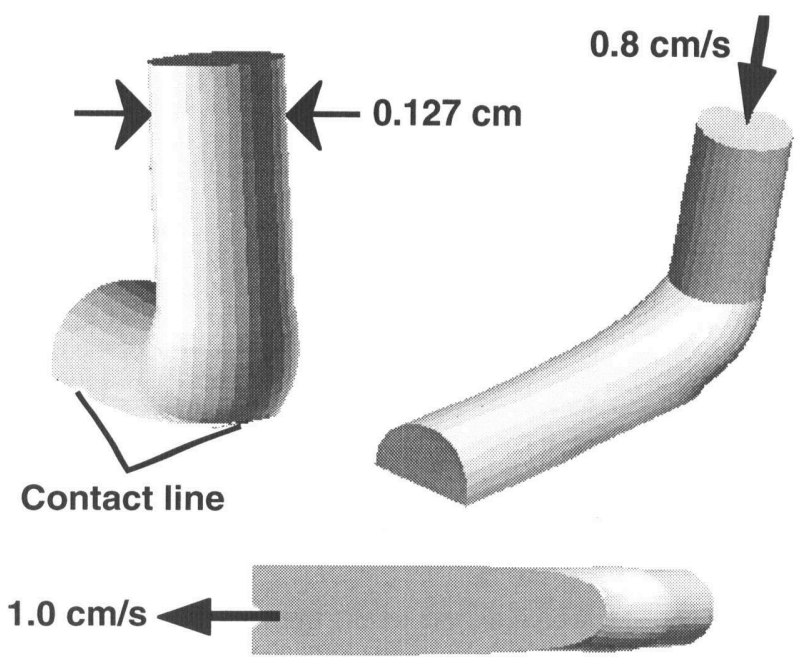

\section{Bottom View}

Figure 2: Three-dimensional modelling of bead flow. Three views of the same Newtonian bead laydown solution. The fluid enters the cylindrical nozzle at $0.8 \mathrm{~cm} / \mathrm{s}$ and the web is moving at $1.0 \mathrm{~cm} / \mathrm{s}$.
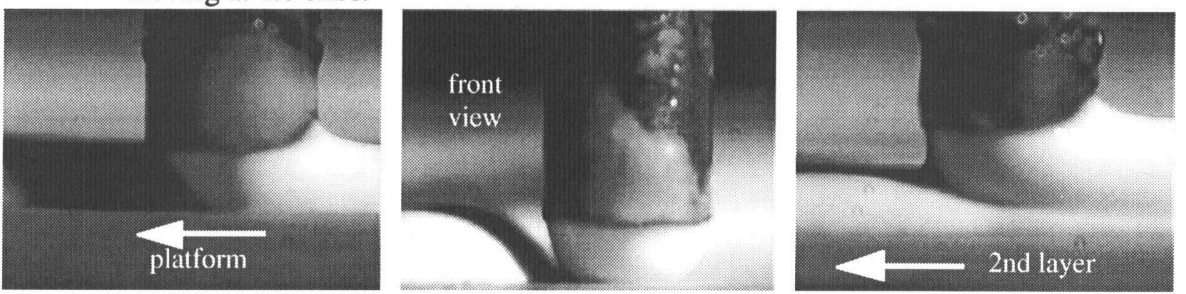

Figure 3: Images of an alumina slurry being deposited onto a moving platform (left and center) and the slurry being deposited onto a previously deposited alumina bead (right).
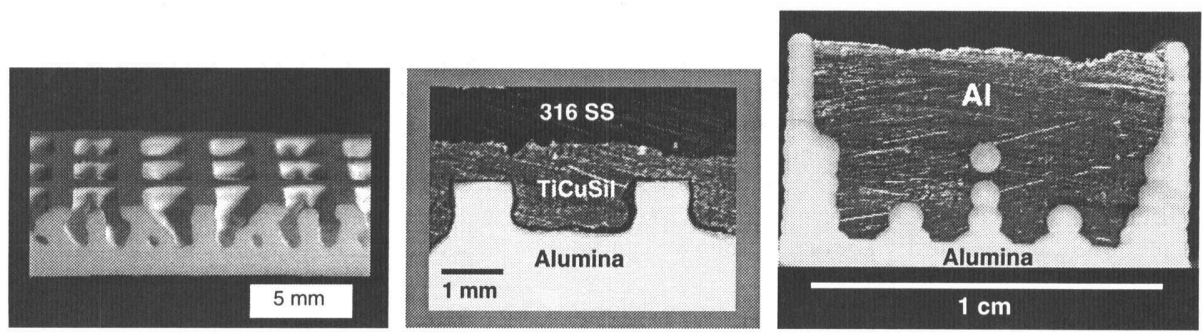

Figure 4: Robocast alumina preforms infiltrated with metal form graded interlocking composites. 


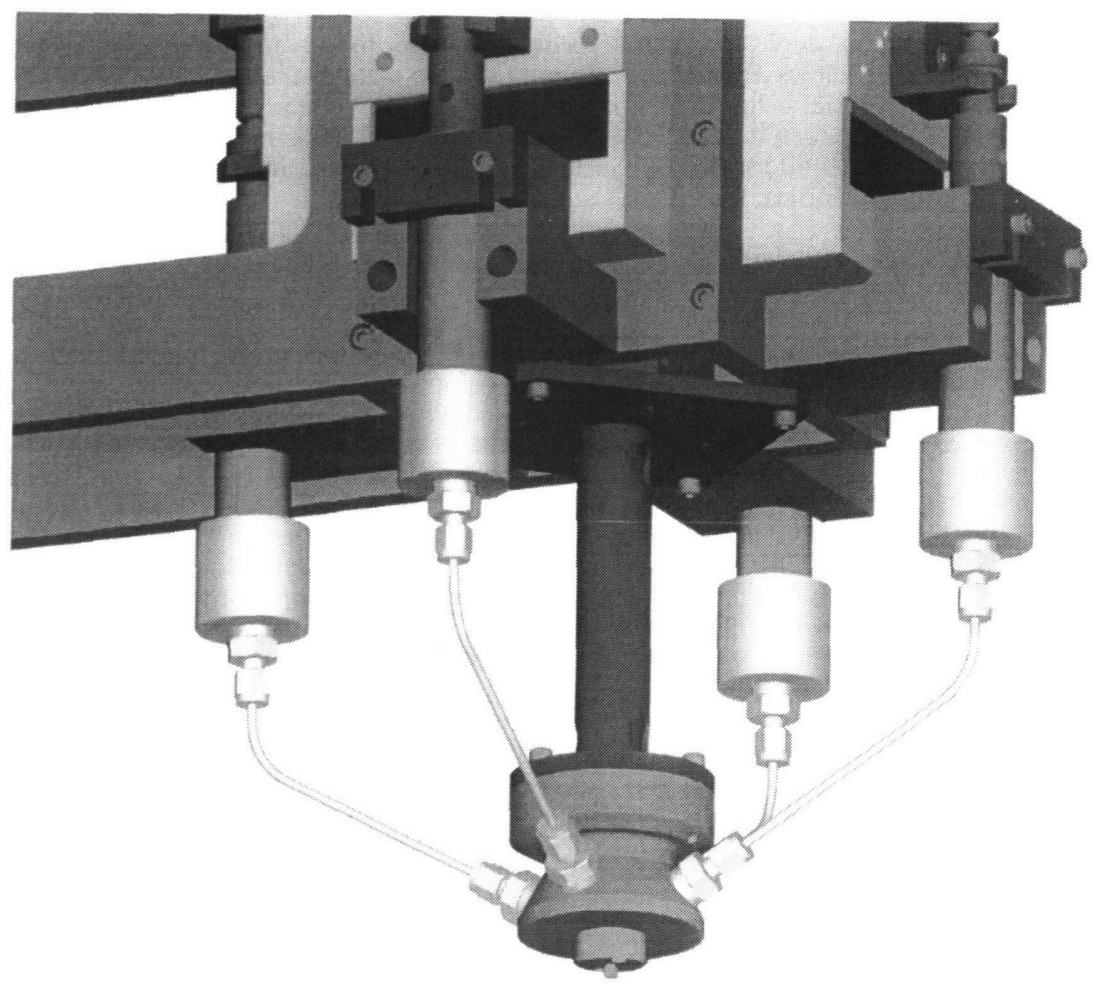

Figure 5: Mixing head capable of depositing four slurries simultaneously.

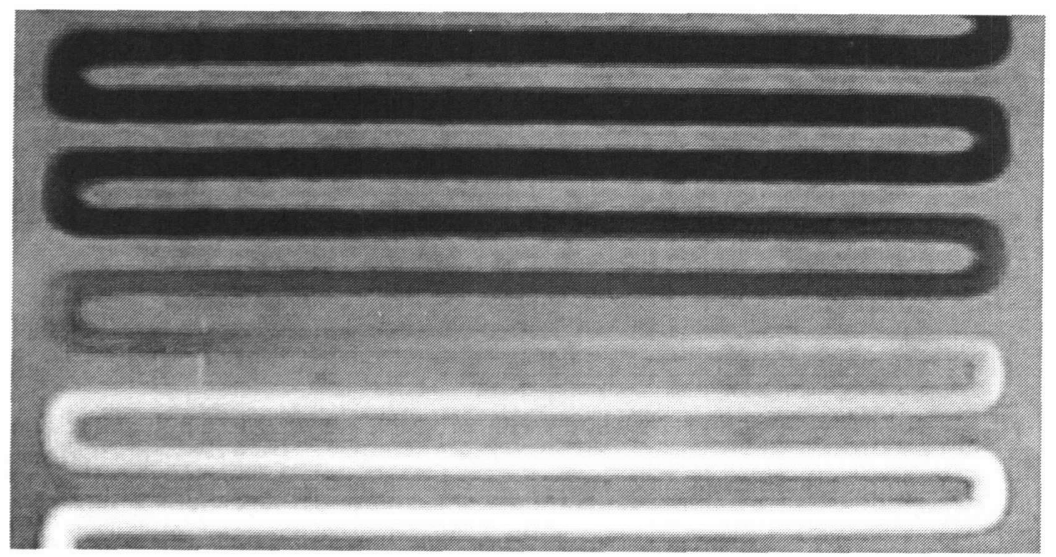

Figure 6: Demonstration of a graded transition between two slurries (bead width $1.5 \mathrm{~mm}$ ). 
- Multimaterial Deposition: The ability to deposit more than one material simultaneously through a single orifice is a recent advance for robocasting that should increase its versatility. Figure 5 shows a schematic of a mixing head that has the capability to dispense up to four different materials. Just before the orifice there is a miniature mixing chamber with a $3 \mathrm{~mm}$ rotatable paddle. When it is desired to deposit ratios of various materials the mixer can be turned on to ensure that a uniform mixture is dispensed through the orifice. For separate and discrete placement of materials, the mixer is automatically turned off.

Figure 6 shows a visual example of complete grading between two materials. This experiment was completed on a dual feed mixing head with a bead width of approximately 1.5 $\mathrm{mm}$ and shows a gradual 100 percent transition from one material to the other. Additionally, in order to build truly three dimensional parts with overhangs, hidden features, and/or buried materials, robocasting must also have the capability to deposit multimaterials discretely. This capability is demonstrated in Fig. 7 for a part fabricated with a horizontal channel that is four bead widths wide. The part in Fig. 7 is made from a kaolin slurry using a fugitive support material. During the build the fugitive material supports the top two layers of kaolin. During drying the fugitive material deforms and is pulled into a thin layer by the surrounding kaolin matrix. During binder burnout and sintering the fugitive material is decomposed and the kaolin densifies. In conclusion, it was determined that for a fabrication technique such as robocasting (i.e., one in which liquid wicking and drying are part of the solidification process) an ideal fugitive material must have the following properties: 1 ) very low solids and organic contents; 2) a high enough yield strength to be an adequate supporting platform; and, 3) a low enough yield stress to deform during wicking and drying without disrupting neighboring materials.

Conceptually, this technique worked very well; however, a crack developed during binder burnout and can be seen on the left hand side of Fig. 7C. It is believed that the build parameters were slightly errant during the first layer of deposition for the fugitive material and some of the material was squeezed on top of the neighboring kaolin bead. This resulted in the crack upon fugitive decomposition. This experiment highlights the need for a knowledge-based ability to precisely predict the shape of deposited beads for all kinds of slurries. Also, there is a need to incorporate sensor controlled feedback for optimum adjustment of build parameters in real time.

Finally, Table I is included to show the current list of materials systems that have been made into robocasting slurries. Some have already been robocasted into samples and others are actively in development.
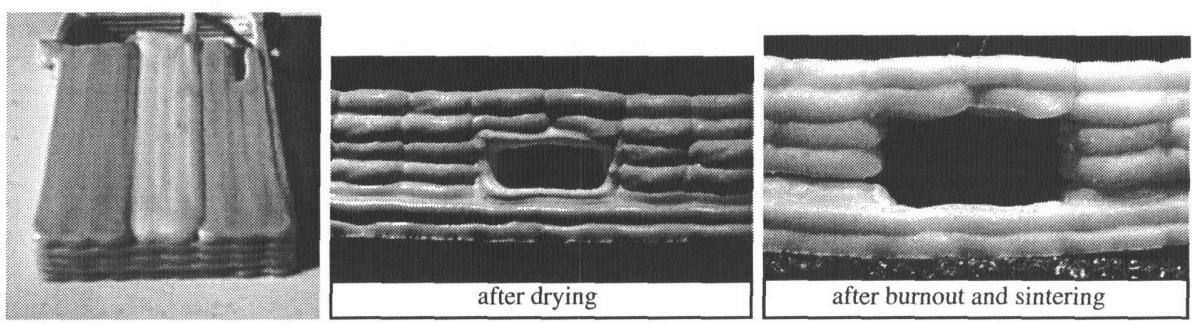

Figure 7: Robocasting a kaolin slurry along with a fugitive material to demonstrate how truly three dimensional parts may be freeformed. Both slurries were deposited with a dual feed mixer through a single orifice. 
Table I

A Current list of materials systems used with robocasting.

\author{
Alumina (dense and porous) \\ $\mathrm{Al}_{2} \mathrm{O}_{3} / \mathrm{TiCuSil}$ composites \\ $\mathrm{Al}_{2} \mathrm{O}_{3} / \mathrm{Al}$ composites \\ $\mathrm{Al}_{2} \mathrm{O}_{3} / \mathrm{Mo}$
}

Thick film pastes, polymers, epoxy

In Development:
PZT

$\mathrm{ZnO}$

Kaolin

Stabilized Zirconia

Mullite

Silicon Nitride, PMN

\section{ACKNOWLEDGMENT}

The following individuals are greatfully acknowledged for their technical contributions and insightful discussions: Prof. Paul Calvert, Bruce King, Hugh Denham, Sherry Morissette, Bruce Tuttle, Thomas Baer, Eric Schlienger, Prof. Jennifer Lewis, Cory Tafoya, Michelle Griffith, Lane Harwell, Prof. Deidre Hirschfeld, and John Stuecker.

Sandia is a multiprogram laboratory operated by Sandia Corporation, a Lockheed Martin Company, for the United States Department of Energy under Contract DE-AC04-94AL85000.

\section{REFERENCES}

1) Cesarano, Baer, and Calvert, Proceedings of the Solid Freeform Fabrication Symposium, Austin, TX, (1997) pp. 25 - 32.

2) Cesarano and Aksay, J. Amer. Ceram. Soc., (1988), 71, 12, 1062-67.

3) Denham, Cesarano, King, and Calvert, "Mechanical Behavior of Robocast Alumina," Proceedings of the Solid Freeform Fabrication Symposium, Austin, TX (1998).

4) King, Morrisette, Denham, Cesarano, and Dimos, "The Influence of Rheology on Deposition Behavior of Slurry-Based Direct Fabrication Systems," Proceedings of the Solid Freeform Fabrication Symposium, Austin, TX (1998).

5) Griffith, Keicher, Atwood, Romero, Smugeresky, Harwell, and Greene, Proceedings of the Solid Freeform Fabrication Symposium, Austin, TX, (1996) p. 125. 